\title{
Magnetic resonance enterography for the evaluation of the deep small intestine in Crohn's disease
}

\author{
Kazuo Ohtsuka ${ }^{1}$, Kento Takenaka $^{1}$, Yoshio Kitazume ${ }^{2}$, Toshimitsu Fujii ${ }^{1}$, Katsuyoshi Matsuoka ${ }^{1}$, Masakazu \\ Nagahori ${ }^{1}$, Maiko Kimura ${ }^{1}$, Takashi Nagaishi $^{1}$, Mamoru Watanabe $^{1}$ \\ ${ }^{1}$ Department of Gastroenterology and Hepatology, Tokyo Medical and Dental University, ${ }^{2}$ Department of Radiology, Tokyo Medical and Dental \\ University, Tokyo, Japan
}

For the control of Crohn's disease (CD) a thorough assessment of the small intestine is essential; several modalities may be utilized, with cross-sectional imaging being important. Magnetic resonance (MR) enterography, i.e., MRE is recommended as a modality with the highest accuracy for CD lesions. MRE and MR enteroclysis are the two methods performed following distension of the small intestine. MRE has sensitivity and specificity comparable to computed tomography enterography (CTE); although images obtained using MRE are less clear compared with CTE, MRE does not expose the patient to radiation and is superior for soft-tissue contrast. Furthermore, it can assess not only static but also dynamic and functional imaging and reveals signs of CD, such as abscess, comb sign, fat edema, fistula, lymph node enhancement, less motility, mucosal lesions, stricture, and wall enhancement. Several indices of inflammatory changes and intestinal damage have been proposed for objective evaluation. Recently, diffusion-weighted imaging has been proposed, which does not need bowel preparation and contrast enhancement. Comprehension of the characteristics of MRE and other modalities is important for better management of CD.

(Intest Res 2016;14:120-126)

Key Words: Magnetic resonance enterography; Computed tomography enterography; Capsule endoscopy; Balloon-assisted enteroscopy; Diffusion magnetic resonance imaging

\section{INTRODUCTION}

Appropriate therapy for a disease is based on its precise diagnosis and assessment. CD is a long-standing chronic IBD. With the recent advent of effective medications, "mucosal healing" is considered as the target of therapy for IBD;" this requires detailed disease assessment on the basis of not only clinical symptoms but also the findings of imaging modalities.

Received March 9, 2016. Revised March 10, 2016.

Accepted March 10, 2016.

Correspondence to Kazuo Ohtsuka, Department of Gastroenterology and Hepatology, Tokyo Medical and Dental University, 1-5-45 Yushima, Bunkyoku, Tokyo 113-8519, Japan. Tel: +81-3-5803-5877, Fax: +81-3-5803-0268, E-mail: kohtsuka.gast@tmd.ac.jp

Financial support: This study was supported in part by Grants-in-Aid from the Japanese Ministry of Education, Culture, Sports, Science and Technology; Health and Labour, Sciences Research Grants for research on intractable diseases from the Ministry of Health, Labour and Welfare of Japan. Conflict of interest: None.
CD may affect any portion of the gastrointestinal tract. Three-quarters of patients with CD have small intestinal lesions. ${ }^{2}$ However, due to the difficulties of endoscopic or cross-sectional approaches for imaging the small intestine, assessment of CD was mainly based on clinical symptoms use of scoring systems, such as the CDAI. Recent progress in modalities, such as capsule endoscopy (CE), ${ }^{3}$ balloonassisted endoscopy (BAE) ${ }^{4}$ as well as cross-sectional imaging, such as CT enterography (CTE) and magnetic resonance (MR) enterography (MRE) enables direct assessment of the lesions deep within the small intestine that cannot be accessed by standard ileocolonoscopy. The cross-sectional imaging can acquire the information of not only the mucosal lesion but also the inflammation in the deep layers of the bowel wall and extraluminal complications, such as abscess and fistula. ${ }^{5}$

Hence, the joint European Crohn's and Colitis Organiza-

๑ Copyright 2016. Korean Association for the Study of Intestinal Diseases. All rights reserved.

This is an Open Access article distributed under the terms of the Creative Commons Attribution Non-Commercial License (http://creativecommons.org/licenses/by-nc/4.0)

which permits unrestricted non-commercial use, distribution, and reproduction in any medium, provided the original work is properly cited. 
tion (ECCO)/European Society of Gastrointestinal and Abdominal Radiology (ESGAR) guidelines in 2013 mentioned that MRE and CTE are important cross-sectional imaging techniques for the assessment of the small intestine. ${ }^{6}$ These modalities are regarded as having the highest accuracy for evaluation of CD lesions. MRE is an excellent tool in diagnosing and monitoring CD in its daily course. ${ }^{7}$ Recently, this journal reviewed CTE and MRE; ${ }^{8}$ here, the recent advances in MRE for patients with small intestinal CD are reviewed.

\section{MRE AND MR ENTEROCLYSIS}

MRI, because of higher resolution and faster acquisition of images, has now become the modality of choice for imaging the small intestine. Contrasts are used for better imaging of the intestinal wall. Adequate distension of the small intestine is also important for images with good-quality and for accuracy of diagnosis.

There are two methods of imaging that fully distend the intestine. One is MR enteroclysis, in which the contrast is administered through a nasojejunal tube, and the other is MRE, in which the contrast is administered orally without tubing. ${ }^{9}$ Several studies compared enteroclysis to MRE. ${ }^{10-12}$ Proximal small bowel distension is frequently less optimal in MRE than in MR enteroclysis. However, both methods are equivalent for ileum distension and clinical information. MR enteroclysis requires insertion of a nasojejunal tube, which is often performed under fluoroscopy and, therefore, exposes the patient to ionizing radiation. Additionally, patients often find insertion of the tube to be unpleasant and even painful. ${ }^{13}$ $\mathrm{CD}$ is a chronic disease that requires repeated examinations, and evading MR examinations due to an uncomfortable procedure may impede precise monitoring, especially in younger patients. MR enteroclysis is often not chosen as the first-line MRI because it has no overwhelming advantages compared with MRE. Many institutes prefer oral administration of contrast, especially for pediatric and adolescent patients; ${ }^{14}$ we also use only MRE.

\section{MRE AND CTE}

On comparing MRE with CTE, both modalities show similar sensitivity and specificity. A recent meta-analysis of 290 patients with CD from 6 different studies showed that the pooled sensitivity and specificity for MRE in detecting active small bowel CD were $87.9 \%$ and $81.2 \%$, respectively, with a weighted area under the curve (AUC) of 0.905 . The pooled sensitivity and specificity of CTE in detecting active small bowel CD was $85.8 \%$ and $83.6 \%$, respectively, with an AUC of 0.898. AUCs of MRE in detecting fistula, stenosis, and abscess were $0.936,0.931$, and 0.996 , respectively, compared with $0.963,0.616$, and 0.899 for CTE. ${ }^{15}$ It was concluded that MRE is comparable to CTE and is a good alternative for evaluation of patients with CD. Patients with CD were frequently evaluated with imaging, such as CT and other radiological modalities during acute exacerbations. ${ }^{16}$ Reducing the radiation exposure, as much as possible, is recommended; therefore, MRE has become the standard imaging modality at many pediatric institutions because it does not use ionizing radiation that may increase the possibility of malignancy, particularly in younger patients. ${ }^{17}$ To this end, currently, the American College of Radiology recommends using both CTE and MRE for imaging of children and young adults with known CD and an acute exacerbation, but recommends using only MRE for the patients with stable, non-acute mild symptoms of CD. ${ }^{18}$

Another advantage of MRE over CTE is that it can acquire bowel images at multiple time-points and cinematic images to evaluate peristalsis. This cine MRI provides functional information of bowel movement. The visualization of peristalsis helps in the assessment of the bowel adhesions, fistula, and stenosis. Contrary to CTE, MRE provides both anatomic and functional information. ${ }^{19} \mathrm{MRE}$ also has better soft-tissue contrast resolution compared with CT and is superior in detecting fibrosis. It can help characterize bowel wall tissue composition and plan therapies based on this information. Newly developed medications, such as biologics, are very effective in treating inflammatory lesions. However, fibrotic strictures are not responsive to medical therapies; they require mechanical treatments, such as balloon dilation, strictureplasty, and bowel resection. ${ }^{20}$

There are some limitations of MRE compared with CT. CT has better spatial resolution and requires a significantly shorter acquisition time. Several sequences are used for MRE; therefore, longer examination time is needed. MRE is currently less accessible and significantly more costly, ${ }^{21}$ and as a result, it is not used as often as other imaging modalities.

\section{ASSESSMENT OF DISEASE}

\section{Findings of CD Using MRE}

Several signs of inflammation and intestinal damage in the evaluation of CD can be assessed using MRE, such as abscess, comb sign, fat edema, fistula, lymph node enhancement, reduced motility, mucosal lesions, strictures, and 
wall enhancement. A meta-analysis showed that the most important signs of inflammation are wall thickness and wall T2-hyperintensity. Sensitivities were $>90 \%$ at the patient level and $>65 \%$ at the bowel segment level. Motility is also a highly sensitive sign. The specific parameters were wall T2-hyperintensity and mucosal lesions, such as ulcers, fissuring, cobble-stoning, and pseudopolyps. The specificities are $>90 \%$ at the patient level and $>95 \%$ at the bowel segment level. As for intestinal damage, abscess, stricture, and fistula had consistently high sensitivity and specificity, especially at the patient level. ${ }^{22}$

MRE can detect lesions by providing images with high quality and reproducibility, even in a global multicenter setting. ${ }^{23}$ It facilitates more objective assessment of disease and will be used as an important indicator for therapeutic intervention trial in future.

\section{Assessment of Inflammation}

Scoring of disease activity is important for objective assessment, and several indices for this have been proposed. The most widely used MRE score is the MR index of activity (MaRIA). ${ }^{24}$ It is calculated as $1.5 \times$ bowel wall thickness in millimeters $(\mathrm{mm})+0.02 \times$ relative contrast enhancement +5 $\times$ edema $+10 \times$ ulceration. MaRIA is strongly correlated with the Crohn's disease endoscopic index of severity (CDEIS).

Contrast enhancement of the bowel wall is necessary to calculate MaRIA. However, after removing the parameter relative contrast enhancement, which requires contrast enhancement, the modified formula $(1.5 \times$ wall thickening $+5 \times$ intramural edema $+10 \times$ ulceration) was comparable to MaRIA in the evaluation of disease activity in CD, with significant correlation. Therefore, MRE can be used without contrast enhancement for patients who are intolerant to gadolinium-based contrast or those with severe renal failure. ${ }^{25}$ The global MaRIA score was calculated as the sum of the MaRIA in ileum, five colorectal segments: ascending colon, transverse colon, descending colon, sigmoid, and rectum. ${ }^{26}$ However, allocation for the small bowel is relatively small; thus, Applied MaRIA can been used by dividing the small intestine into three segments: terminal ileum, proximal ileum, and jejunum. Applied MaRIA is well correlated to findings of $\mathrm{BAE}^{27}$

The other proposed scoring system, Crohn's disease activity score (CDAS), is based on mural thickness and T2 signal on fat-saturated imaging. CDAS score is based on the following calculation: $1.79+1.34 \times$ mural thickness $+0.94 \times$ mural T2 score ${ }^{28}$ CDAS showed a sensitivity of $81 \%$, specificity of
$70 \%$, and AUC of 0.77 for predicting acute inflammation and was modified as a global assessment score called the MRE global score (MEGS), which is a sum of scores of qualitative and semiquantitative grading of bowel wall thickness, mural and peri-mural T2 signal, $\mathrm{T} 1$ enhancement, mural-enhanced pattern length of the diseased segment, and extramural features. ${ }^{29}$ The bowel was divided into nine segments and each segment scored independently. The small bowel was divided into jejunum, ileum, and terminal ileum.

\section{Assessment of Intestinal Damage}

The clinical manifestations of CD consist of inflammation and accumulated intestinal damage. The Lémann index was proposed for the assessment of intestinal damage. ${ }^{30}$ It assesses the upper tract, small bowel, colon/rectum, and anus, with three grades. The small bowel is divided into $20-\mathrm{cm}$ segments. There are investigational methods designated for each area of the gastrointestinal tract. Strictures of the upper tract and the colon are assessed using endoscopy or MRI/CT. However, the small bowel is investigated using MRI/CT only. Direct comparison between MRE and enteroscopy revealed less sensitivity of MRE for strictures. ${ }^{31}$ Evaluation of MRE in cases of surgery showed similar results. ${ }^{32}$ Preoperative MRE for 43 patients with CD showed that sensitivity was $68 \%$ for stenosis and $60 \%$ for fistula. The negative predictive values of MRE were $65 \%$ for stenosis and $81 \%$ for fistula. MRE had $78 \%$ accuracy for stenosis and $85 \%$ for fistula. False-negative rates for MRE were $40 \%$ for fistula and $32 \%$ for stenosis. Assessment of stricture is important because it is a major cause of surgery in $\mathrm{CD}$, although endoscopic balloon dilatation is effective in some cases. ${ }^{33}$ Further research on the efficacy of investigational methods is needed.

\section{Assessment of Therapeutic Effect}

Several effective medicines for CD, such as anti-tumor necrosis factor (anti-TNF) antibody, have emerged. They are often costly, and the choice of treatment is sometimes difficult. MRE can assess current inflammation effectively and may guide the therapeutic decision. Some MRE findings will help predict outcome following commencement of anti-TNF antibody therapy. The strictures and penetrating complications detected using MRE often require future surgery. ${ }^{34}$ MRE is a useful modality in monitoring the efficacy of treatment. Transmural and healing detected using MRE correlates with changes in CD clinical activity during antiTNF therapy ${ }^{35}$ Bowel motility assessed using MRE is also 
observed with response to anti-TNF antibody therapy. MRE may detect non-responsiveness to anti-TNF during early phases of treatment. ${ }^{36}$

Preoperative assessment of disease is crucial for better surgical outcomes. Laparoscopic surgery is commonly used for colorectal cancer resection, and its utility is increasing in the field of IBD. However, conversion from laparoscopic surgery to open laparotomy is not rare in IBD; the conversion results in longer operative courses, increased resource utilization, and increased morbidity than the complete laparoscopic and open surgery from the beginning. Unexpected intraoperative findings, such as large inflammatory mass, adhesions, ileosigmoid fistula, and pelvic abscess, are the causes of conversion. Cross-sectional imaging provides adequate information of disease extent and, thus, will help avoid conversion. ${ }^{37}$ A retrospective analysis of 187 laparoscopic surgeries with preoperative CTE/NRE shows a negative correlation to conversion. However, the ability of MRE to detect stenosis is limited. ${ }^{31}$

\section{COMPARISON TO OTHER MODALITIES}

\section{CE}

CE noninvasively visualizes the entire small intestinal mucosa. A comparison between MRE and CE reveled that $\mathrm{CE}$ is more sensitive for detection of lesions at previously unrecognized locations, and MRE is superior for detection of the location of lesions. ${ }^{38}$ The limitation of CE is retention due to strictures, which are often observed in CD; MRE may help predict such strictures. ${ }^{39}$ However, it is recommended that investigation with a patency capsule should be undertaken before CE because the detection of strictures by MRE is only moderate.

\section{BAE}

It enables detailed, direct observation of mucosa; however, the invasiveness of endoscopy should not be ignored. The findings of MRE and ileocolonoscopy were compared; their inflammatory findings were correlated well. ${ }^{26} \mathrm{~A}$ direct comparison between MRE and enteroscopy in 100 patients with $\mathrm{CD}$ has been reported. MRE detected ulcerative lesions in the small intestine with $82.4 \%$ sensitivity and $87.6 \%$ specificity. It detected major strictures with $58.8 \%$ sensitivity and $90.0 \%$ specificity. It was concluded that MRE had enough sensitivity for detecting active lesions; however, it was less sensitive in detecting intestinal damage, such as strictures. ${ }^{31}$

\section{Ultrasonography}

Ultrasonography (US) is a sensitive modality for diagnosis and evaluation of disease activity and is comparable to MRI US is widely available and non-invasive; however, its accuracy depends on the examiner and is low in the proximal to terminal ileum region. ${ }^{40} \mathrm{~A}$ comparison between US and MRE in 29 pediatric patients with CD showed an almost perfect agreement for abscess, substantial for maximum bowel wall thickness, stricture and penetrating disease. ${ }^{41} \mathrm{~A}$ comparison in 120 adults showed comparable sensitivities and specificities for US and MRE. ${ }^{42}$ The concordance of CD location was high; there was fair concordance in the detection of strictures and abscesses. However, US was less accurate compared with MRE for determining the extent of disease. MRE was more accurate for the detection of penetrating lesions, particularly in the deep pelvic region. A multicenter, non-randomized, single-arm, prospective comparison study is ongoing. ${ }^{43}$ Further studies will clarify the proper use of such non-ionizing radiation techniques.

\section{NEW TECHNIQUES}

MRE uses several sequences and the technique is varied. Standardization enables comparison and assessment of techniques and facilitates the uptake of MRE. Recently, consensus for the optimal technique for MRE in CD was released with following recommendations: $:^{44}$ patient preparation (fasting 2-4 hours prior to the procedure); oral contrast ( $>900$ cc biphasic enteric contrast administered in divided doses 45-60 minutes prior to scanning); intravenous contrast (single dose of $0.1 \mathrm{mmol} / \mathrm{kg}$ extracellular space gadolinium agent or gadobenate dimeglumine injected at $\geq 2 \mathrm{cc} /$ s); spasmolytics (recommended); coverage (from small bowel to perineum); required pulse sequences (T2-weighted sequence-axial and coronal, one plane with fat saturation, dynamic contrast-enhanced coronal T1-weighted fast spin three-dimensional gradient-echo-acquire at 45 seconds with two subsequent coronal acquisitions, axial contrastenhanced T1-weighted fast spin three-dimensional gradientecho); and contraindications for intravenous contrast (for noncontrast MRE, additional pulse sequences, such as diffusion and cine sequences may be added to T2-weighted imaging.

\section{Diffusion-Weighted Imaging}

Diffusion-weighted imaging MRE (DWI-MRE) does not 
need bowel preparation and contrast enhancement. It is useful for patients who cannot receive contrast due to renal failure, pregnancy, or allergy. An early report on DWI-MR colonography reported that it was comparable to gadolinium enhancement for the detection of inflammation in $\mathrm{CD}^{45}$

However, a recent meta-analysis of 1,515 bowel segments for diagnosis and 1,066 bowel segments for assessment of inflammatory severity showed sensitivity and specificity to be $92.9 \%$ and $91 \%$, respectively. ${ }^{46}$ It was concluded that DWIMRE accuracy was heterogeneous among studies and was likely overestimated in some studies. On the other hand, an association between lower apparent diffusion coefficient (ADC) values and the presence of stricture on MRE were reported. A study of 5 pediatric patients with CD showed that ADC values were high in normal segments, moderate in segments with acute inflammation, and low in the presence of fibrosis. ${ }^{47}$ There was a progressive decrease in ADC values during the clinical course. A longitudinal observation of 13 pediatric patients showed that ADC values changed with disease course. ${ }^{48} \mathrm{ADC}$ values of non-fibrostenotic imaging phenotypes increased, although those of fibrostenotic lesions did not change. These reports show the possibility of using DWI-MRE for the assessment of not only inflammation but also fibrosis. MR without contrast is not enough to replace MRE, and further studies are awaited.

\section{Cine Motility}

MRE can provide a quantitative assessment of small bowel motility. The motility of inflamed bowel segments decreases compared with non-inflamed segments. Hence, the decrease in motility can be used as a marker of inflammation. Furthermore, there are advantages of using cine MRE over static imaging to investigate the intestinal damage, such as adhesion, fistula, and stricture. Cine imaging does not require additional patient preparation and only short time is required for image acquisition and reading. There is a report comparing cine MRE to endoscopy in 28 patients with CD. Motility index was calculated and was significantly related to histological activity in the terminal ileum. ${ }^{49}$ In 91 patients with stricturing CD, small bowel motility as indicated on MRE was shown to differ significantly among normal, prestricture, and strictured bowels. ${ }^{50}$ There was a negative correlation between the prestricture bowel diameter and motility.

The 2013 ECCO/ESGAR guidelines mention that the inclusion of small bowel motility evaluation may increase lesion detection rate compared with static MRE alone. ${ }^{5}$ The consensus statement from the Society of Abdominal Radiol- ogy (SAR) Crohn's disease-focused panel published in 2015 mentioned that cine MRE can provide functional evaluation of small bowel peristalsis. ${ }^{44}$

\section{Magnetization Transfer MRI}

Magnetization transfer (MT) was proposed for detection of bowel fibrosis. ${ }^{51}$ The MT ratio was significantly increased in the bowel wall with fibrotic scarring, although it was slightly decreased in the segments with acute inflammation. MT imaging may be useful for distinguishing inflammatory and fibrotic stenosis. Further research is needed to establish the value of MT MRI.

\section{CONCLUSIONS}

The progress of MRE has changed the assessment of CD. Complete utilization of the recent advances in therapeutics requires more objective assessment and detailed information of the affected organs. The deep small intestine is a major segment affected by CD. Before the advent of MRE, it was difficult to assess the deep small intestine. MRE can provide information on the deep small intestine and extraluminal manifestations of CD. It helps monitor the effect of medicines and guides procedures for surgical treatment. MRE is not a mere substitute for CT, as non-ionizing radiation cross sectional imaging modality; it changes the static imaging into objective dynamic imaging. It is not restricted to anatomic diagnosis, but instead provides a wider, functional analysis.

\section{REFERENCES}

1. Neurath MF, Travis SP. Mucosal healing in inflammatory bowel diseases: a systematic review. Gut 2012;61:1619-1635.

2. Ng SC, Tang W, Ching JY, et al. Incidence and phenotype of inflammatory bowel disease based on results from the Asiapacific Crohn's and colitis epidemiology study. Gastroenterology 2013;145:158-165.

3. Iddan G, Meron G, Glukhovsky A, Swain P. Wireless capsule endoscopy. Nature 2000;405:417.

4. Yamamoto H, Sekine Y, Sato Y, et al. Total enteroscopy with a nonsurgical steerable double-balloon method. Gastrointest Endosc 2001;53:216-220.

5. Cheriyan DG, Slattery E, McDermott S, et al. Impact of magnetic resonance enterography in the management of small bowel Crohn's disease. Eur J Gastroenterol Hepatol 2013;25:550-555. 
6. Panes J, Bouhnik Y, Reinisch W, et al. Imaging techniques for assessment of inflammatory bowel disease: joint ECCO and ESGAR evidence-based consensus guidelines. J Crohns Colitis 2013;7:556-585.

7. Lang G, Schmiegel W, Nicolas V, Brechmann T. Impact of small bowel MRI in routine clinical practice on staging of Crohn's disease. J Crohns Colitis 2015;9:784-794.

8. Kim SH. Computed tomography enterography and magnetic resonance enterography in the diagnosis of Crohn's disease. Intest Res 2015;13:27-38.

9. Masselli G, Casciani E, Polettini E, Gualdi G. Comparison of MR enteroclysis with MR enterography and conventional enteroclysis in patients with Crohn's disease. Eur Radiol 2008;18:438447.

10. Schreyer AG, Geissler A, Albrich H, et al. Abdominal MRI after enteroclysis or with oral contrast in patients with suspected or proven Crohn's disease. Clin Gastroenterol Hepatol 2004;2:491-497.

11. Negaard A, Paulsen V, Sandvik L, et al. A prospective randomized comparison between two MRI studies of the small bowel in Crohn's disease, the oral contrast method and MR enteroclysis. Eur Radiol 2007;17:2294-2301.

12. Lawrance IC, Welman CJ, Shipman P, Murray K. Small bowel MRI enteroclysis or follow through: which is optimal? World J Gastroenterol 2009;15:5300-5306.

13. Negaard A, Sandvik L, Berstad AE, et al. MRI of the small bowel with oral contrast or nasojejunal intubation in Crohn's disease: randomized comparison of patient acceptance. Scand J Gastroenterol 2008;43:44-51.

14. Ognibene NM, Basile M, Di Maurizio M, Petrillo G, De Filippi C. Features and perspectives of MR enterography for pediatric Crohn disease assessment [published online ahead of print February 2, 2016]. Radiol Med. doi:10.1007/s11547-015-0613-2.

15. Qiu Y, Mao R, Chen BL, et al. Systematic review with meta-analysis: magnetic resonance enterography vs. computed tomography enterography for evaluating disease activity in small bowel Crohn's disease. Aliment Pharmacol Ther 2014;40:134-146.

16. Desmond AN, O’Regan K, Curran C, et al. Crohn's disease: factors associated with exposure to high levels of diagnostic radiation. Gut 2008;57:1524-1529.

17. Towbin AJ, Sullivan J, Denson LA, Wallihan DB, Podberesky DJ. CT and MR enterography in children and adolescents with inflammatory bowel disease. Radiographics 2013;33:1843-1860.

18. Duigenan S, Gee MS. Imaging of pediatric patients with inflammatory bowel disease. AJR Am J Roentgenol 2012;199:907-915.

19. Heye T, Stein D, Antolovic D, Dueck M, Kauczor HU, Hosch W. Evaluation of bowel peristalsis by dynamic cine MRI: detection of relevant functional disturbances-initial experience. J Magn Reson Imaging 2012;35:859-867.
20. Quencer KB, Nimkin K, Mino-Kenudson M, Gee MS. Detecting active inflammation and fibrosis in pediatric Crohn's disease: prospective evaluation of MR-E and CT-E. Abdom Imaging 2013;38:705-713.

21. Amitai MM, Ben-Horin S, Eliakim R, Kopylov U. Magnetic resonance enterography in Crohn's disease: a guide to common imaging manifestations for the IBD physician. J Crohns Colitis 2013;7:603-615

22. Church PC, Turner D, Feldman BM, et al. Systematic review with meta-analysis: magnetic resonance enterography signs for the detection of inflammation and intestinal damage in Crohn's disease. Aliment Pharmacol Ther 2015;41:153-166.

23. Coimbra AJ, Rimola J, O'Byrne S, et al. Magnetic resonance enterography is feasible and reliable in multicenter clinical trials in patients with Crohn's disease, and may help select subjects with active inflammation. Aliment Pharmacol Ther 2016;43:6172 .

24. Rimola J, Rodriguez S, García-Bosch O, et al. Magnetic resonance for assessment of disease activity and severity in ileocolonic Crohn's disease. Gut 2009;58:1113-1120.

25. Scardapane A, Ambrosi A, Salinaro E, et al. Assessment of disease activity in small bowel Crohn's disease: comparison between endoscopy and magnetic resonance enterography using MRIA and modified MRIA score. Gastroenterol Res Pract 2015;2015:159641. doi: 10.1155/2015/159641.

26. Ordás I, Rimola J, Rodríguez S, et al. Accuracy of magnetic resonance enterography in assessing response to therapy and mucosal healing in patients with Crohn's disease. Gastroenterology 2014;146:374-382.

27. Takenaka K, Ohtsuka K, Kitazume Y, et al. Correlation of the endoscopic and magnetic resonance scoring systems in the deep small intestine in Crohn's disease. Inflamm Bowel Dis 2015;21:1832-1838.

28. Steward MJ, Punwani S, Proctor I, et al. Non-perforating small bowel Crohn's disease assessed by MRI enterography: derivation and histopathological validation of an MR-based activity index. Eur J Radiol 2012;81:2080-2088.

29. Makanyanga JC, Pendsé D, Dikaios N, et al. Evaluation of Crohn's disease activity: initial validation of a magnetic resonance enterography global score (MEGS) against faecal calprotectin. Eur Radiol 2014;24:277-287.

30. Pariente B, Mary JY, Danese S, et al. Development of the Lémann index to assess digestive tract damage in patients with Crohn's disease. Gastroenterology 2015;148:52-63.

31. Takenaka K, Ohtsuka K, Kitazume Y, et al. Comparison of magnetic resonance and balloon enteroscopic examination of the small intestine in patients with Crohn's disease. Gastroenterology 2014;147:334-342. 
32. Seastedt KP, Trencheva K, Michelassi F, et al. Accuracy of CT enterography and magnetic resonance enterography imaging to detect lesions preoperatively in patients undergoing surgery for Crohn's disease. Dis Colon Rectum 2014;57:1364-1370.

33. Hirai F, Beppu T, Takatsu N, et al. Long-term outcome of endoscopic balloon dilation for small bowel strictures in patients with Crohn's disease. Dig Endosc 2014;26:545-551.

34. Gibson DJ, Murphy DJ, Smyth AE, et al. Magnetic resonance enterography findings as predictors of clinical outcome following antitumor necrosis factor treatment in small bowel Crohn's disease. Eur J Gastroenterol Hepatol 2015;27:956-962.

35. Eder P, Katulska K, Krela-Kaźmierczak I, et al. The influence of anti-TNF therapy on the magnetic resonance enterographic parameters of Crohn's disease activity. Abdom Imaging 2015;40:2210-2218.

36. Plumb AA, Menys A, Russo E, et al. Magnetic resonance imaging-quantified small bowel motility is a sensitive marker of response to medical therapy in Crohn's disease. Aliment Pharmacol Ther 2015;42:343-355.

37. Mino JS, Gandhi NS, Stocchi LL, et al. Preoperative risk factors and radiographic findings predictive of laparoscopic conversion to open procedures in Crohn's disease. J Gastrointest Surg 2015;19:1007-1014.

38. Greener T, Klang E, Yablecovitch D, et al. The impact of magnetic resonance enterography and capsule endoscopy on the reclassification of disease in patients with known Crohn's disease: a prospective Israeli IBD Research Nucleus (IIRN) study [published online ahead of print January 8, 2016]. J Crohns Colitis. doi: http://dx.doi.org/10.1093/ecco-jcc/jjw006.

39. Rozendorn N, Klang E, Lahat A, et al. Prediction of patency capsule retention in known Crohn's disease patients by using magnetic resonance imaging. Gastrointest Endosc 2016;83:182-187.

40. Panés J, Bouzas R, Chaparro M, et al. Systematic review: the use of ultrasonography, computed tomography and magnetic resonance imaging for the diagnosis, assessment of activity and abdominal complications of Crohn's disease. Aliment Pharmacol Ther 2011;34:125-145.

41. Dillman JR, Smith EA, Sanchez R, et al. Prospective cohort study of ultrasound-ultrasound and ultrasound-MR enterography agreement in the evaluation of pediatric small bowel Crohn disease. Pediatr Radiol 2016;46:490-497.

42. Castiglione F, Mainenti PP, De Palma GD, et al. Noninvasive diagnosis of small bowel Crohn's disease: direct comparison of bowel sonography and magnetic resonance enterography. Inflamm Bowel Dis 2013;19:991-998.
43. Taylor S, Mallett S, Bhatnagar G, et al. METRIC (MREnterography or ulTRasound in Crohn's disease): a study protocol for a multicentre, non-randomised, single-arm, prospective comparison study of magnetic resonance enterography and small bowel ultrasound compared to a reference standard in those aged 16 and over. BMC Gastroenterol 2014;14:142. doi: 10.1186/1471-230X-14-142.

44. Grand DJ, Guglielmo FF, Al-Hawary MM. MR enterography in Crohn's disease: current consensus on optimal imaging technique and future advances from the SAR Crohn's diseasefocused panel. Abdom Imaging 2015;40:953-964.

45. Oussalah A, Laurent V, Bruot O, et al. Diffusion-weighted magnetic resonance without bowel preparation for detecting colonic inflammation in inflammatory bowel disease. Gut 2010;59:1056-1065.

46. Choi SH, Kim KW, Lee JY, Kim KJ, Park SH. Diffusion-weighted magnetic resonance enterography for evaluating bowel inflammation in Crohn's disease: a systematic review and metaanalysis. Inflamm Bowel Dis 2016;22:669-679.

47. Kovanlikaya A, Beneck D, Rose M, et al. Quantitative apparent diffusion coefficient (ADC) values as an imaging biomarker for fibrosis in pediatric Crohn's disease: preliminary experience. Abdom Imaging 2015;40:1068-1074.

48. Rosenbaum DG, Rose ML, Solomon AB, Giambrone AE, Kovanlikaya A. Longitudinal diffusion-weighted imaging changes in children with small bowel Crohn's disease: preliminary experience. Abdom Imaging 2015;40:1075-1080.

49. Menys A, Atkinson D, Odille F, et al. Quantified terminal ileal motility during MR enterography as a potential biomarker of Crohn's disease activity: a preliminary study. Eur Radiol 2012;22:2494-2501.

50. Menys A, Helbren E, Makanyanga J, et al. Small bowel strictures in Crohn's disease: a quantitative investigation of intestinal motility using MR enterography. Neurogastroenterol Motil 2013;25:967-e775[975].

51. Pazahr S, Blume I, Frei P, et al. Magnetization transfer for the assessment of bowel fibrosis in patients with Crohn's disease: initial experience. MAGMA 2013;26:291-301. 